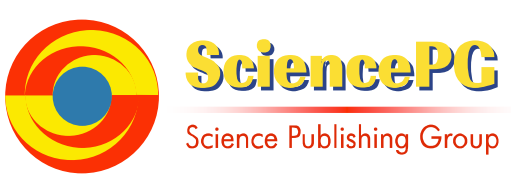

\title{
Preparation on Chitosan Derivative Nanoparticles as Prolonged Releasing Drug Carrier
}

\author{
Bohua Feng, Liufen Peng* \\ College of Medical Information Engineering, Guangdong Pharmaceutical University, Guangzhou, P. R. China
}

Email address:

tfengbh@163.com (Liufen Peng)

${ }^{*}$ Corresponding author

To cite this article:

Bohua Feng, Liufen Peng. Preparation on Chitosan Derivative Nanoparticles as Prolonged Releasing Drug Carrier. International Journal of Biomedical Science and Engineering. Vol. 4, No. 3, 2016, pp. 28-33. doi: 10.11648/j.ijbse.20160403.13

Received: August 30, 2016; Accepted: September 10, 2016; Published: September 27, 2016

\begin{abstract}
Ricinoleic acid grafted amphiphilic carboxymethy chitosan (CMC-g-RA) was synthetised as a carrier to load botanical drug rotenone (Rot). Then Rot/CMC-g-RA water dispersion in nanoscale was prepared, whose shape, zeta potential, loading efficiency and outdoor stability were characterized accordingly. The results indicated that the sizes, polydispersity index, zeta potential of Rot/CMC-g-RA particles were affected by concentrations of this water dispersion. When the ratio of carrier to drug ascended, the water dispersion had monodisperse nanoparticle sizes with negative charge on nanoparticle surface. And the water dispersion restrained Rot degradation in natural environment with higher loading efficiency.
\end{abstract}

Keywords: Nanocarrier, Chitosan Derivative, Drug Carrier

\section{Introduction}

Water dispersion pesticide formulation is known as an environmentally friendly formulation. Most lipid-soluble drugs could be solubilized in water depending on various surfactants, and it is considered that polymers surfactants are superior to micromolecule surfactants in many respects. The polymers surfactants with hydrophobic groups on polymer chains could have much better loading capacity of pestcide, which would improve water dispersion's stability and elongate the drug releasing time. Furthermore, biodegradability of polymers surfactants would be beneficial to environmental protection. Chitosan is known as biodegradable alkaline polysaccharides, having pharmacological activity and growth regulation for crops [1]. The self aggregation of the chitosan derivative modified by hydrophobic group, which indicated the modified chitosan could be a good carrier for lipid-soluble drugs [2].

Carboxymethy chitosan grafted ricinoleic acid (CMC-g-RA, figure 1), a new chitosan derivative, had be synthesized in our early research [3]. Both hydrophobic long carbon chain (ricinoleic acid, RA) and hydrophilic carboxymethy were grafted in chitosan chain, where RA was well known as a natural green pesticide. Therefore, CMC-g-RA had the capability of forming self assembly polymer micelles, where lipid-soluble pesticide could be solubilized. However lipid soluble pesticides were conventionally dispersed in highly toxic solvent and additives to produce formulations such as emulsion, emulsifiable concentrate, suspension concentrate and so on. The particles sizes in above formulations were usually more than $1 \mu \mathrm{m}$. Attributed to the large surface area effect and small size effect of nanoparticles [4], particles sizes in nanoscale were beneficial to improving bioavailability of pesticide. In this study the well known botanical and lipid-soluble pesticide rotenone [5] would be carried by CMC-g-RA. The Rot degradation could be restrained with protection of carrier, which would be evaluated in natural environment compared with micromolecule carrier RA-Na. The controlling approaches to the performance of Rot/CMC-g-RA water dispersion would be also discussed, where particles sizes, polydispersity index (PDI), zeta potential, morphological structure, components concentrations relations and loading efficiency (LE) were demonstrated in details. 


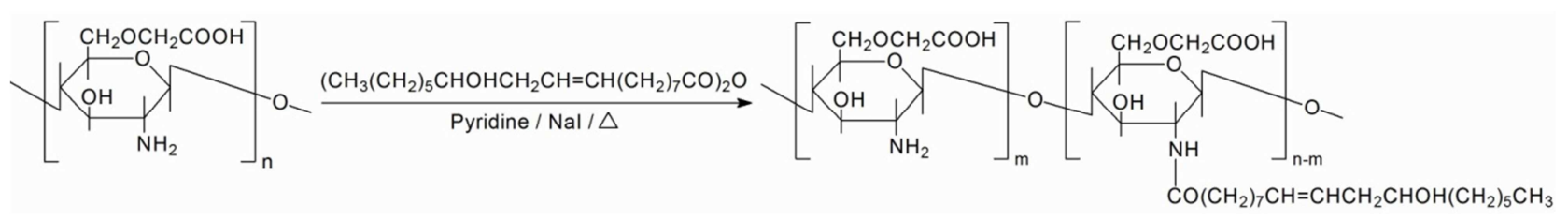

Figure 1. Preparation of CMC-g-RA [3].

\section{Experimental}

\subsection{Materials}

CMC-g-RA、RA-Na were prepared by polymers laboratory of department of materials Science and Engineering in Jinan University (Guangzhou, China), rotenone standard was purchased from Sigma (USA), and rotenone was obtained from Jiaxing fuli chemistry factory $(98 \%$, Fengshun of Guangdong, China).

\subsection{Devices}

EM (XL-30E, Philips, Netherland), FTIR (Equi-nox55, Bruk, Germany), ZetaPALS (Brookhaven, USA), Elemental Analyzer (EA2400II, Perkin-El-mer, USA), Sprayer (QH-003, Qihua Plastic Fact-ory), HPLC(1100, Agilent, USA), Supercentrifuge (3K30, Sartorius-Sigma Laboratory Centrifuges, Germany), Freeze drier(FD1.0-60E, Heto, Denmark).

\subsection{Preparation of Rot/CMC-g-RA Water Dispersion}

The samples of CMC-g-RA were dissolved in deionized water to obtain carrier solution $(10 \mathrm{mg} / \mathrm{ml})$. The Rot solution was then blent with carrier solution to form Rot/CMC-g-RA water dispersions, where the Rot concentrations were from 0.20 to $0.20 \mathrm{mg} / \mathrm{ml}$ separately.

\subsection{Characterization of Rot/CMC-g-RA Water Dispersion}

Ultracentrifugation of Rot/CMC-g-RA was implemented for $30 \min \left(25,000 \mathrm{rpm}, 4^{\circ} \mathrm{C}\right)$, and the precipitation was collected for freeze drying. The ATR-FTIR spectrograms of Rot/CMC-g-RA particles, CMC-g-RA and Rot were then measured. The particles sizes, PDIs and zeta potentials were investigated with the ZetaPALS correspondingly. The particles morphological structures were demonstrated by SEM after dropping and drying the solutions on base chips. Unloading drug concentrations in the supernatant of Rot/CMC-g-RA solution $(1.5 \mathrm{ml})$ and LE were respectively measured and calculated by HPLC method [6], by which chromatographic column $250 \mathrm{~mm} \times 4.0 \mathrm{~mm}, \mathrm{C} 18$ reversed-phase column, column temperature $30^{\circ} \mathrm{C}$, mobile phase volume ratio methanol: water $=75: 15$, flow velocity 1.0 $\mathrm{ml} / \mathrm{min}$, wavelength $281 \mathrm{~nm}$ and sampling volume $15 \mu \mathrm{l}$ were followed.

\subsection{Outdoor Stability}

Rot/CMC-g-RA water dispersion was first prepared by Rot
$(0.10 \mathrm{mg} / \mathrm{ml})$ and CMC-g-RA $(0.50 \mathrm{mg} / \mathrm{ml})$. Then control groups prepared by small molecule surfactant $\mathrm{RA}-\mathrm{Na}$ and water/acetone solution were obtained with the same Rot concentrations. After nylon sheets (size $20 \mathrm{~cm} \times 20 \mathrm{~cm}$ per sheet) were immersed in the water dispersion and control groups respectively. All sheets were dried outdoor under sun from 1 to 11 days, which were put into methanol to leach the Rot left respectively every 2 days. The Rot concentrations were measured by HPLC referring to previous study [6].

\section{Results and Discussion}

\subsection{Rot/CMC-g-RA FTIR Spectroscopy Analysis}

FTIR spectrograms of CMC-g-RA particles, Rot/CMC-g-RA and Rot were separately shown in figure 2.

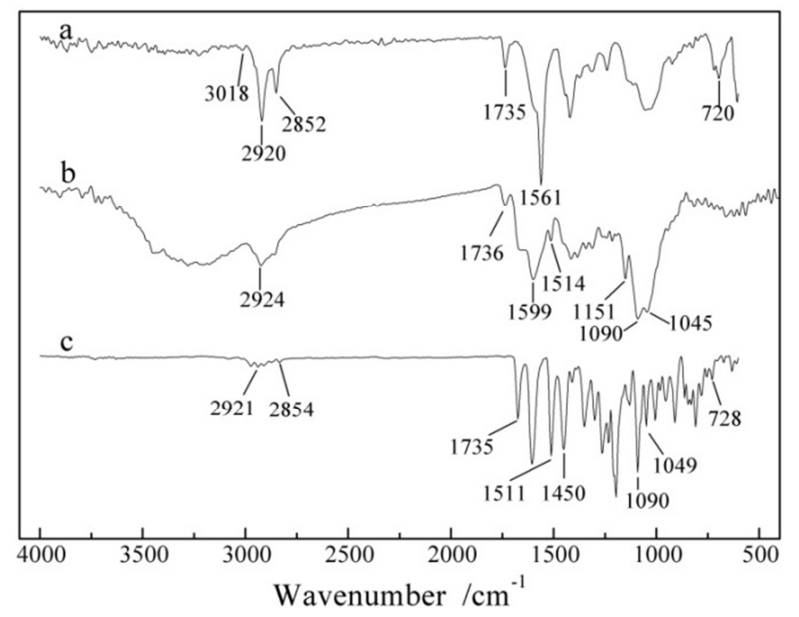

Figure 2. FTIR spectrums of CMC-g-RA(a), Rot/CMC-g-RA(b) and Rot(c).

In the CMC-g-RA spectrogram plot (figure 2a), substitution reaction happened on $-\mathrm{NH}_{2}$ groups, therefore the adsorption peak of $-\mathrm{NH}_{2}$ moved from $1597 \mathrm{~cm}^{-1}$ to $1560 \mathrm{~cm}^{-1}$. The peak at $1735 \mathrm{~cm}^{-1}$ standed for amide carbonyl made by the reaction. The characteristic absorption peak of the $\mathrm{C}=\mathrm{C}$ appeared at $3018 \mathrm{~cm}^{-1}$, and C-H stretching vibration peak at $2920 \mathrm{~cm}^{-1}$ and $2852 \mathrm{~cm}^{-1}$ became sharp and strong. The peak appeared around $720 \mathrm{~cm}^{-1}$ was observed, which was known as $-\left(\mathrm{CH}_{2}\right)_{\mathrm{n}}$ adsorption peak $(n>4)$ of the RA. In the Rot/CMC-g-RA spectrogram (figure $2 \mathrm{~b}$ ), main adsorption peaks appeared in figure $2 \mathrm{a}$ remained while peaks intensity weaken. And obvious peak at $1151 \mathrm{~cm}^{-1}$ demonstrated C-O-C group of the Rot molecule. The above changes indicated Rot was loaded by carrier CMC-g-RA. 


\subsection{Nanoparticles Morphological Structure}

The SEM photos of Rot/CMC-g-RA particles were showed in figure 3. The smooth spherical particles sizes were most less than $500 \mathrm{~nm}$. Hydrophilic carboxymethy trended to water phase and the hydrophobic RA chains inclined towards oil phase to reduce surface tension, which formed shell of particles. Rot could be effectively loaded in the carriers, because hexagonal plate crystalline of Rot was not obviously observed.

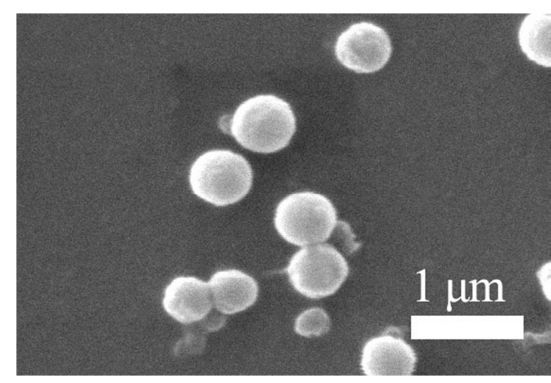

a

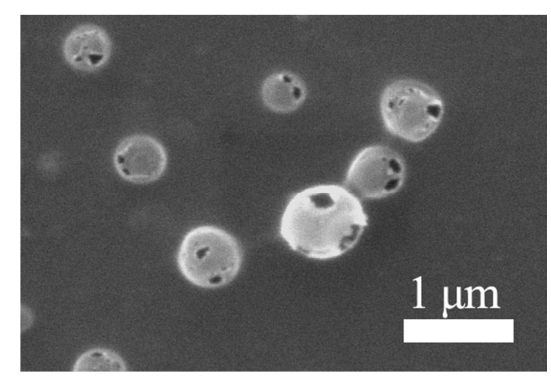

b

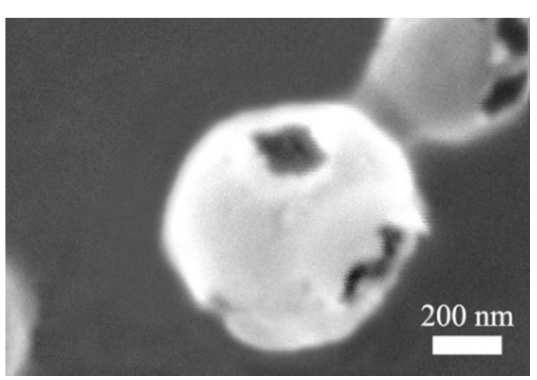

$\mathrm{c}$

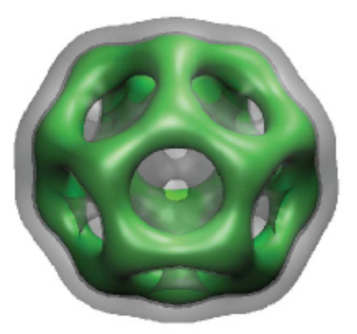

d

Figure 3. SEM photos of Rot / CMC-g-RA nanoparticles, drying at ambient temperature (a); drying at $60{ }^{\circ} \mathrm{C}$ (b) \& (c) where $[C M C$-g- $R A]=0.50 \mathrm{mg} / \mathrm{ml}$, [Rot] $=0.10 \mathrm{mg} / \mathrm{ml}$. And simulation graph of polymers cage-shaped micelle (d), where green and gray respectively represent hydrophobic and hydrophilic segments [7].
Rot/CMC-g-RA particles with porous structure were also obtained (figure $3 \mathrm{~b}$ ) by drying the solution under $60^{\circ} \mathrm{C}$ before SEM observation instead of drying under room temperature. The detail in figure $3 \mathrm{c}$ clearly demonstrated core-shell structure of Rot/CMC-g-RA particle. A model graph according to self-consistent mean field theory (SCMFT) could be drawn like figure $3 \mathrm{~d}$, which showed amphiphilic polymer could form porous cage-shaped particle. It was inferred that the higher drying temperature accelerated the solvent evaporation, thus the shapes of Rot/CMC-g-RA particles in water dispersion were reserved.

\subsection{Effects of Concentration on Nanoparticles Sizes, PDIs and Zeta Potential}

Rot/CMC-g-RA particles sizes, PDIs and zeta potentials were measured by dynamic light scattering (DLS) under variant concentrations of carrier and drug, and the results were listed in table 1. In brief, the particle sizes were between 200 and $400 \mathrm{~nm}$, where the PDIs were less than 0.25 . The smaller the PDIs were, the better the uniformity of particles were [8]. Since carboxymethy groups trended to water phase as expected, zeta potential data in table 1 indicated the surface charge of particles were negative.

In Rot/CMC-g-RA water dispersion, the particles sizes could be regulated due to the varying carrier or drug concentrations. The minimum particle size was $190.2 \mathrm{~nm}$ when lower CMC-g-RA and Rot concentrations were present (figure 4a). CMC-g-RA concentrations were increased from 0.1 to $0.3 \mathrm{mg} / \mathrm{ml}$ coupled with greater particles sizes, while Rot concentrations were kept steadily as shown in figure 4e. Obviously particles sizes enlarged (562.2 $\mathrm{nm})$ also appeared after Rot concentrations were grown to $0.06 \mathrm{mg} / \mathrm{ml}$, while concentrations of CMC-g-RA were invariant.

There was a linear relationship between the solubilizing capability and micelle size that usually increased with the concentrations of polymer carriers [9]. Higher carriers concentrations meant adding more hydrophobic RA groups, which resulted in better compatible between CMC-g-RA and Rot when the Rot concentrations were constant. Meanwhile, Rot would tend to enter hydrophobic domain in CMC-g-RA micelle. Furthermore, according to the Steric stabilization theory, coverage of CMC-g-RA would reduce interfacial energy among Rot and water, which could form more stable water dispersion. The interfacial energy mentioned could also be lower after increasing Rot concentrations while remaining CMC-g-RA concentrations invariable. However, increasing process of particles sizes occurred when the ratio of carrier to drug was less than 10 (figure 5). If CMC-g-RA concentrations proceeded to grow up ([CMC-g-RA] $>0.3 \mathrm{mg} / \mathrm{ml}$ in figure 4 ), the force among micelles, surface molecules of micelle and solution molecules would dramatically increased too. Thus bigger micelles would disaggregate to more smaller ones to decrease free energy of mixing, and particles sizes were reduced correspondingly, shown in figure 5 , where ratio of carrier to drug was more than 10 . 
Table 1. Particles sizes, PDIs and zeta potential under varying concentrations of CMC-g-RA and Rot.

\begin{tabular}{|c|c|c|c|c|}
\hline CMC-g-RA [mg/ml] & Rot $[\mathrm{mg} / \mathrm{ml}]$ & Particles sizes [nm] & PDIs & Zeta potentials [mV] \\
\hline 0.10 & 0.02 & $190.2 \pm 12.3$ & $0.433 \pm 0.002$ & $-58.50 \pm 0.91$ \\
\hline 0.10 & 0.03 & $289.7 \pm 20.5$ & $0.344 \pm 0.002$ & $-55.62 \pm 1.68$ \\
\hline 0.10 & 0.04 & $271.5 \pm 19.9$ & $0.284 \pm 0.002$ & $-40.73 \pm 5.49$ \\
\hline 0.10 & 0.05 & $256.4 \pm 20.0$ & $0.157 \pm 0.002$ & $-50.50 \pm 1.52$ \\
\hline 0.10 & 0.06 & $376.3 \pm 27.3$ & $0.185 \pm 0.003$ & $-45.55 \pm 5.52$ \\
\hline 0.30 & 0.02 & $316.2 \pm 17.5$ & $0.206 \pm 0.002$ & $-36.98 \pm 0.95$ \\
\hline 0.30 & 0.03 & $402.3 \pm 31.0$ & $0.171 \pm 0.001$ & $-63.27 \pm 0.68$ \\
\hline 0.30 & 0.05 & $475.2 \pm 37.2$ & $0.138 \pm 0.004$ & $-60.33 \pm 2.15$ \\
\hline 0.30 & 0.06 & $562.2 \pm 28.2$ & $0.154 \pm 0.001$ & $-58.47 \pm 1.29$ \\
\hline 0.50 & 0.02 & $218.4 \pm 18.1$ & $0.075 \pm 0.001$ & $-28.00 \pm 4.21$ \\
\hline 0.50 & 0.03 & $352.2 \pm 24.8$ & $0.081 \pm 0.001$ & $-60.14 \pm 6.85$ \\
\hline 0.50 & 0.04 & $341.2 \pm 18.6$ & $0.112 \pm 0.001$ & $-56.81 \pm 1.75$ \\
\hline 0.50 & 0.05 & $306.9 \pm 17.5$ & $0.140 \pm 0.001$ & $-69.64 \pm 2.91$ \\
\hline 0.50 & 0.06 & $511.4 \pm 26.7$ & $0.137 \pm 0.001$ & $-52.28 \pm 0.95$ \\
\hline 0.70 & 0.04 & $309.6 \pm 16.1$ & $0.009 \pm 0.000$ & $-65.13 \pm 0.91$ \\
\hline 0.70 & 0.05 & $348.9 \pm 22.3$ & $0.003 \pm 0.001$ & $-74.69 \pm 2.21$ \\
\hline 0.70 & 0.06 & $355.8 \pm 21.1$ & $0.010 \pm 0.001$ & $-70.35 \pm 6.62$ \\
\hline
\end{tabular}

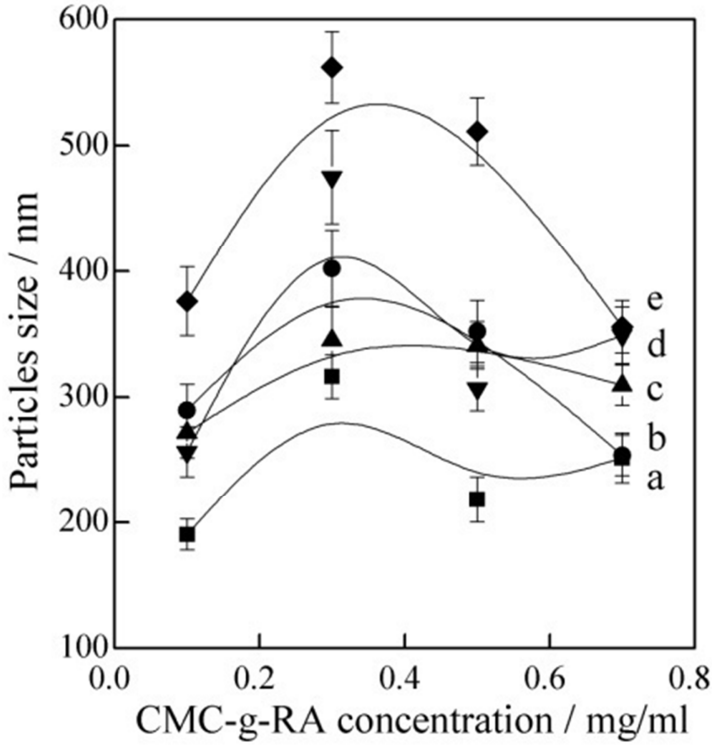

Figure 4. Effect of CMC-g-RA concentrations on particles sizes, a-e: [Rot]= $0.02-0.06 \mathrm{mg} / \mathrm{ml}$.

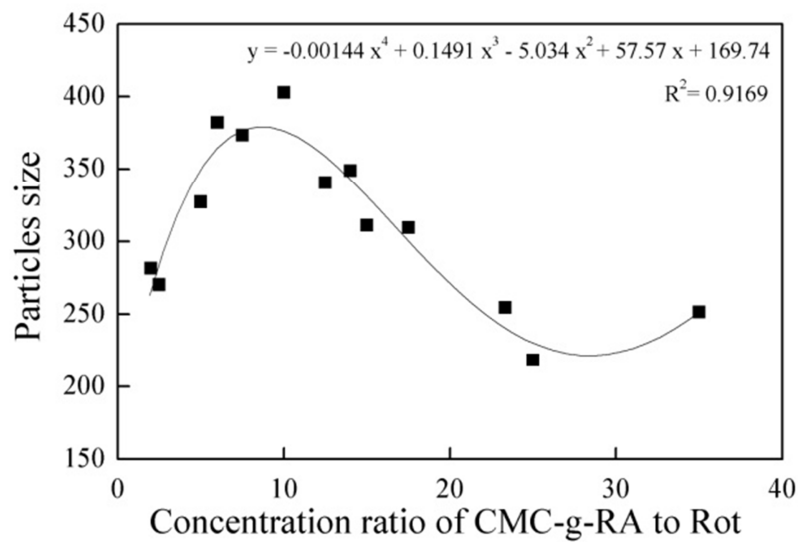

Figure 5. Effect of [CMC-g-RA]/[Rot] on particles sizes.

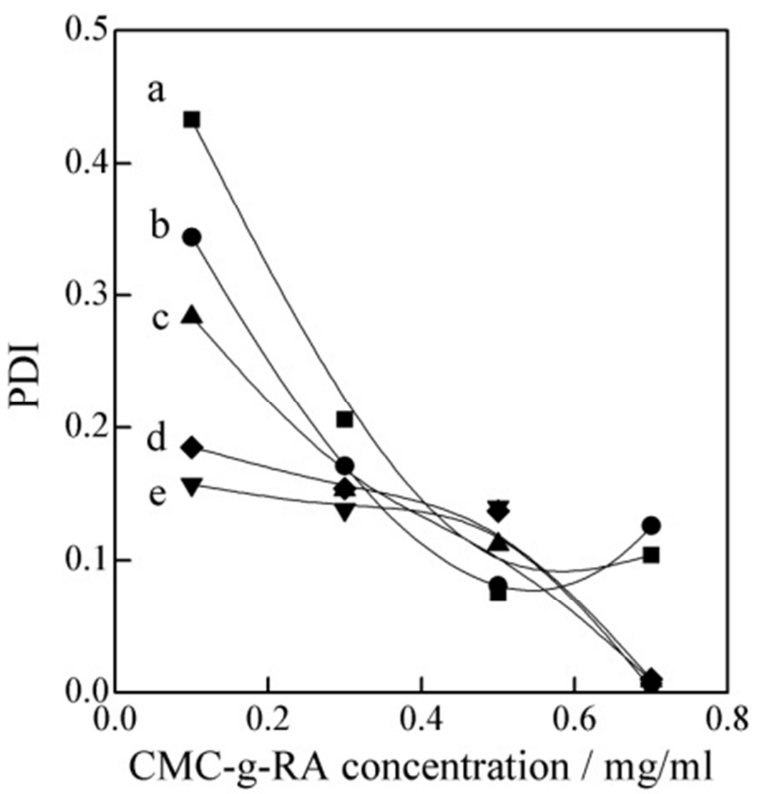

Figure 6. Effect of CMC-g-RA concentrations on PDIs, a-e: [Rot $]=0.02 \sim$ $0.06 \mathrm{mg} / \mathrm{ml}$.

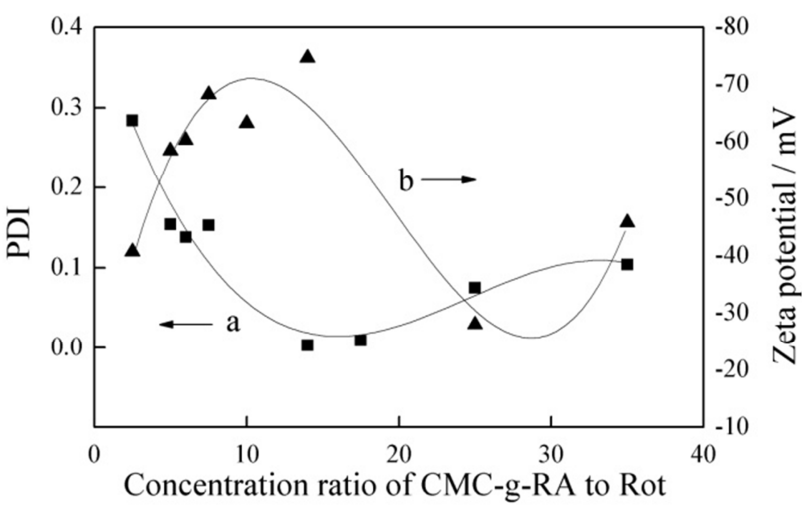

Figure 7. Effect of [CMC-g-RA]/[Rot] on PDIs (a) and Zeta potentials (b). 
Rot/CMC-g-RA particles sizes in nanoscale could be controlled by adjusting concentrations ratio of CMC-g-RA to Rot. The PDIs should be less than 0.25 to unify particles sizes and improve bioavailability of pesticide. As the Rot concentrations remained without change, PDIs became much smaller with CMC-g-RA concentrations raising (figure 6). For example, CMC-g-RA concentrations increased from 0.1 $\mathrm{mg} / \mathrm{ml}$ to $0.5 \mathrm{mg} / \mathrm{ml}$ while PDIs decreased from 0.344 to 0.081 (figure 6b), which was closed to monodisperse of nanoparticles sizes. When ratio of carrier to drug was less than 10 , PDIs decreased rapidly with the ratio growing up shown in figure $7 \mathrm{a}$.

The zeta potentials were also influenced by the concentrations ratios of CMC-g-RA to Rot (figure 7b). Higher zeta potential, usually $>30 \mathrm{mV}$, standed for stronger electrostatic repulsive force that was beneficial to maintain water dispersion steady [10]. When ratio of carrier to drug was less than 15 , zeta potential increased rapidly with the ratio growing up. The maximum was $-74.69 \mathrm{mV}$ as concentration of CMC-g-RA and Rot were $0.70 \mathrm{mg} / \mathrm{ml}$ and $0.05 \mathrm{mg} / \mathrm{ml}$ respectively (table 1). One reason was that more carboxymethy groups with negative charge were added into water dispersion along with increased carriers concentrations. On the other hand, with Rot concentrations grown up, the stronger affinity among hydrophobic groups in carrier and drug also resulted in more carboxymethy groups specifically trended to enter water phase.

According to measuring results of particles sizes, PDIs and zeta potential of Rot/CMC-g-RA water dispersion, stable and uniform nanoparticles water dispersion could be obtained when concentrations ratios of $\mathrm{CMC}-\mathrm{g}-\mathrm{RA}$ to Rot was regulated between 5 and 10 .

\subsection{LE of Rot/CMC-g-RA Nanoparticles}

The Rot standard's retention time was $5.718 \mathrm{~min}$ after HPLC test [7], and loading efficiency (LE) of Rot/CMC-g-RA samples could be calculated by the equation:

$$
\mathrm{LE} \text { of Rot } / \mathrm{CMC}-\mathrm{g}-\mathrm{RA}=\frac{\mathrm{A}-\mathrm{B}}{\mathrm{A}}
$$

where A standed for total Rot concentration and B represented the free Rot concentration in supernatant.

LE firstly increased to $68 \%$ and then decreased to $22 \%$ with CMC-g-RA concentrations increasing (figure 8a), when the Rot concentrations of Rot/CMC-g-RA samples was 0.05 $\mathrm{mg} / \mathrm{ml}$. During this process concentrations ratios of CMC-g-RA to Rot were about 2 to 18 . The similar varying trend was observed in figure 5, the peak value of $\mathrm{LE}$ corresponded to $[\mathrm{CMC}-\mathrm{g}-\mathrm{RA}] /[\mathrm{Rot}]=10$. When Rot concentration was $0.10 \mathrm{mg} / \mathrm{ml}$ (figure $8 \mathrm{~b}$ ), the LE increased from $22 \%$ to $65 \%$ with CMC-g-RA concentrations growing up, where coincided with $[\mathrm{CMC}-\mathrm{g}-\mathrm{RA}] /[\mathrm{Rot}]<10$ in figure 5 .

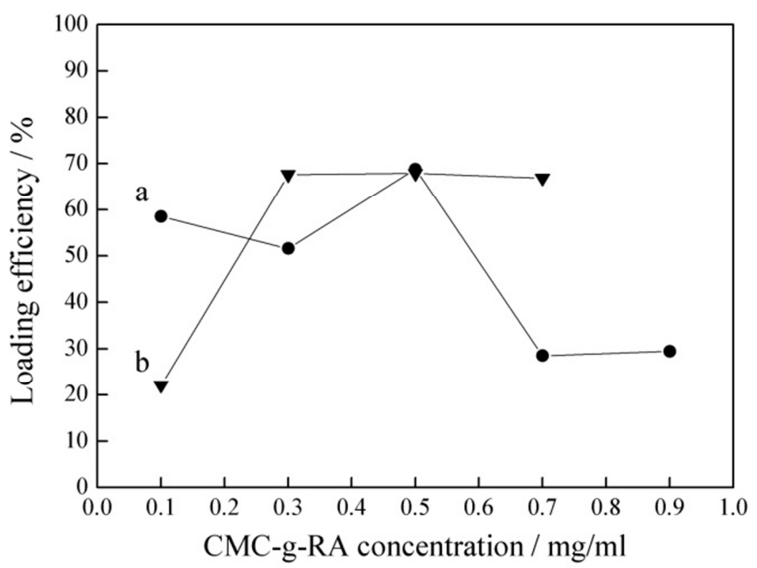

Figure 8. $L E$ of Rot/CMC-g-RA, $[R o t]=0.05 \mathrm{mg} / \mathrm{ml}$ (a) and $[R o t]=0.10$ $m g / m l(b)$.

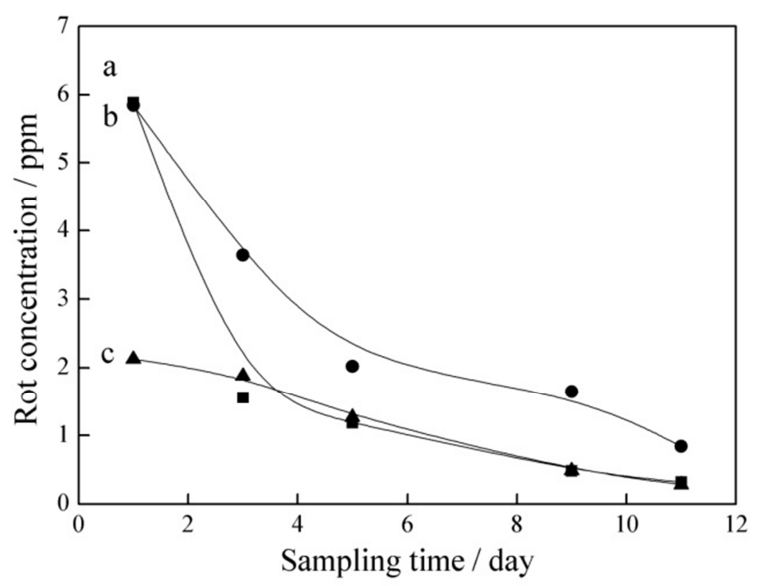

Figure 9. Rot concentrations under sampling times in leaching liquor of Rot/CMC-g-RA (a), Rot/RA-Na (b) and Rot water dispersion (c).

\subsection{Outdoor Stability of Water Dispersion}

Stability of the Rot/CMC-g-RA water dispersion was evaluated by measuring residual Rot concentrations under sampling times in leaching liquor (figure 9). Samples $([\mathrm{CMC}-\mathrm{g}-\mathrm{RA}] /[\mathrm{Rot}]=5,[\mathrm{CMC}-\mathrm{g}-\mathrm{RA}]=0.50 \mathrm{mg} / \mathrm{ml},[\mathrm{Rot}]=$ $0.10 \mathrm{mg} / \mathrm{ml}$ ) with the LE of $68 \%$ (figure $9 \mathrm{~b}$ ) were chosen compared with Rot/RA-Na and Rot water dispersion samples as control groups. During 11 days outdoor testing, the residual Rot concentrations of the samples were higher than control groups. In the first 3 days, Rot concentrations were decreasing from about $5.8 \mathrm{ppm}$ to $3.7 \mathrm{ppm}$, at the same time, control groups curves dropped quickly. After 4 days the residual Rot concentrations remained 2.3-1.1 ppm in samples. Polymer carrier CMC-g-RA efficiently restrained the Rot degradation instead of control groups. High LE implied the unloading Rot molecules were seldom outside the micelle, which would not reduce the Rot concentrations rapidly. The polymer CMC-g-RA also provided much effective protection for Rot comparing with small molecule carrier RA-Na or no carrier in water. Thus carrier CMC-g-RA was benefit to releasing Rot with much stability and higher concentrations. 


\section{Conclusion}

The amphiphilic CMC-g-RA turned to polymeric micelles in water by self-assembly, which became efficient drug carrier for lipid-soluble pesticide Rot. The Rot/CMC-g-RA water dispersion were prepared successfully by blending carrier and drug together. Rot/CMC-g-RA particles were smooth spherical in nanoscale, and this small-size effect could correspondingly improve the infiltration and bioavailability of drug. Adjusting concentrations ratio of CMC-g-RA to Rot, especially $[\mathrm{CMC}-\mathrm{g}-\mathrm{RA}] /[\mathrm{Rot}]=5$ to 10 , could get nanoparticles of smaller, uniform particles sizes and higher zeta potential. Rot was protected efficiently by carriers in micelles, the highest LE of Rot/CMC-g-RA was up to $68 \%$ when $[\mathrm{CMC}-\mathrm{g}-\mathrm{RA}] /[\mathrm{Rot}]=5$. Therefore, Rot was stable releasing during 11 days in environment. This study had given an advice to produce non-toxic drug carrier for solving the solubilization and controlled releasing of lipid-soluble Rot in water, which would contribute to develop a green and efficient nano-botanical pesticide preparing method of water-based formulation. And the insecticidal principle of Rot/CMC-g-RA water dispersion and its performance on improving Rot bioavailability would be discussed in following papers.

\section{Acknowledgments}

The authors would like to thank professor Zi-Yong Zhang for his guidance. This project is supported by Medical Scientific Research Foundation of Guangdong Province, P. R. China (B2014203) and Science \& Technology Planning Project of Guangdong Province, P. R. China (2016A020 215162). The authors also appreciate the Polymers Laboratory in the Department of Materials Science and Engineering at Jinan University for supporting this work.

\section{References}

[1] A. Sathiyanarayanan, and M. Sathiyabama, "Induced chitinase and chitosanase activities in turmeric plants by application of ß-D-glucan nanoparticles," Notulae Scientia Biologicae, vol. 7, no. 3, pp. 295-298, 2015.
[2] M. A. Elgadir , M. S. Uddin, S. Ferdosh, A. Adam, A. J. K. Chowdhury and M. Z. I. Sarker, "Impact of chitosan composites and chitosan nanoparticle composites on various drug delivery systems: a review," Journal of Food \& Drug Analysis, vol. 23, no. 4, pp. 619-629, 2014.

[3] B. H. Feng and L. F. Peng, "Synthesis and characterization of carboxymethyl chitosan carrying ricinoleic functions as an emulsifier for azadirachtin," Carbohydrate Polymers, vol. 88, no. 2, pp. 576-582, 2012.

[4] D. B. Shenoy and G. B. Sukhorukov. "Engineered microcrystals for direct surface modification with layer-by-layer technique for optimized dissolution," European Journal of Pharmaceutics and Biopharmaceutics, vol. 58, no.3, pp. 521-527, 2004.

[5] A. Kamari, N. F. A. Aljafree and S. N. M. Yusoff, "Oleoyl-carboxymethyl chitosan as a new carrier agent for the rotenone pesticide," Environmental Chemistry Letters, vol. 14, no. 3, pp. 417-422, 2016.

[6] Y. K. Wu, J. Lin and M.Ye, "Determination of Rotenone by High Performance Liquid Chromatography," Yunnan Chemical Technology, vol. 33, no. 2, pp. 59-60, 2006.

[7] X. H. He and F, Schmid, "Spontaneous formation of complex micelles from a homogeneous solution," Physical review letters, vol. 100, no. 13, pp. 137802. 2007.

[8] F. Bassyouni, N. Elhalwany, M. A. Rehim and M. Neyfeh, "Advances and new technologies applied in controlled drug delivery system," Research on Chemical Intermediates, vol. 41, no. 4, pp. 1-36, 2013.

[9] T. Chen, X. H. Zhang and R. Guo, "Surface Activity and Aggregation of Chitosan," Acta Physico-chimica Sinica, vol. 16, no. 11, pp. 1039-1042, 2000.

[10] L. LI, X. Y. Xu and J. P. Zhou, "Preparation and characterization of N-octyl-N'-succinyl chitosan micelles," Chinese Journal of New Drugs, vol. 16, no. 7, pp. 543-547, 2007. 